\title{
THE QSO - ULTRALUMINOUS INFRARED GALAXIES CONNECTION
}

\author{
Sylvain Veilleux \\ Department of Astronomy, University of Maryland \\ veilleux@astro.umd.edu \\ Dong-Chan Kim \\ Institute of Astronomy and Astrophysics, Academia Sinica \\ kim@asiaa.sinica.edu.tw \\ David B. Sanders \\ Institute for Astronomy, University of Hawaii \\ sanders@galileo.ifa.hawaii.edu
}

\begin{abstract}
For the past several years, our group has pursued a vigorous groundbased program aimed at understanding the nature of ultraluminous infrared galaxies. We recently published the results from a optical/nearinfrared spectroscopic survey of a large statistically complete sample of ultraluminous infrared galaxies (the "IRAS 1-Jy sample"). We now present the results from our recently completed optical/near-infrared imaging survey of the 1-Jy sample. These data provide detailed morphological information on both large scale (e.g., intensity and color profiles, intensity and size of tidal tails and bridges, etc) and small scale (e.g., nuclear separation, presence of bars, etc) that helps us constrain the initial conditions necessary to produce galaxies with such high level of star formation and/or AGN activity. The nature of the interdependence between some key spectroscopic and morphological parameters in our objects (e.g., dominant energy source: super-starburst versus quasar, nuclear separation, merger phase, star formation rate, and infrared luminosity and color) is used to clarify the connection between starbursts, ultraluminous infrared galaxies, and quasars.
\end{abstract}

Keywords: QSOs, Seyfert galaxies, starburst galaxies, origin, evolution 


\section{INTRODUCTION}

Ultraluminous infrared galaxies (ULIGs; $\log \left[\mathrm{L}_{\mathrm{IR}} / \mathrm{L}_{\odot}\right] \geq 12$ by definition) may provide the clearest observational link between galaxy mergers, starbursts and powerful AGN. However, the exact nature of ULIGs remains unclear. The most important questions, and the ones we propose to address here, are: (1) What is the dominant energy source in ULIGs (starburst versus AGN)?, and (2) Is there a evolutionary connection between ULIGs and quasars?

In recent years, several surveys to faint flux levels in the IRAS database have been carried out to search for luminous objects. Arguably the most important of these studies is the ' 1 Jy' survey of Kim (1995). This study provides a complete list of the brightest ULIGs with $\mathrm{F}[60 \mu \mathrm{m}]>1 \mathrm{Jy}$ which is not biased toward 'warm' quasar-like objects with large $\mathrm{F}[25$ $\mu \mathrm{m}] / \mathrm{F}[60 \mu \mathrm{m}]$ ratios. The ' 1 Jy' sample contains 118 objects with $z=$ $0.02-0.27$ and $\log \left[\mathrm{L}_{\mathrm{ir}} / \mathrm{L}_{\odot}\right]=12.00-12.84$. The infrared luminosities of these objects therefore truly overlap with the bolometric luminosities of optical quasars. Other surveys have discovered objects of comparable luminosity at fainter flux levels as well as a few 'hyperluminous' objects at higher $\mathrm{L}_{\mathrm{ir}}$. However, the ' 1 Jy' sample contains the brightest objects at a given luminosity, hence the best candidates for follow-up studies.

The results of our analysis of the IRAS database on the ' 1 Jy' sample were published in Kim \& Sanders (1998) and are discussed in these proceedings (Sanders contribution). The present paper summarizes the results from our ground-based follow-up surveys. In Section 2, we discuss the results from our optical and near-infrared spectroscopy of this sample, and in Section 3, the preliminary results from our optical and near-infrared imaging survey. Section 4 summarizes our conclusions.

\section{OPTICAL AND INFRARED SPECTROSCOPY OF THE 1 JY SAMPLE}

The results from our optical and near-infrared spectroscopic surveys were published recently in Veilleux, Kim, \& Sanders (1999a) and Veilleux, Sanders, \& Kim (1999b), respectively. The main conclusions of this analysis are the following:

1. The fraction of luminous infrared galaxies with Seyfert characteristics increases rapidly with increasing $L_{\text {ir }}$. About $30 \%$ of the ULIGs host a Seyfert 1 or Seyfert 2 nucleus. For $L_{\text {ir }}>10^{12.3} L_{\odot}$, this fraction is nearly $50 \%$.

2. From $50 \%$ to $70 \%$ of the Seyfert $2 \mathrm{~s}$ in our sample show signs of an AGN in the near-infrared (e.g., broad-line region or strong [Si VI] 
$1.962 \mu \mathrm{m})$, therefore confirming the detection of genuine AGN in these objects. In contrast, none of the optically classified LINERs and H II galaxies in our near-infrared sample shows any obvious signs of an energetically important AGN.

3. Combining our optical and near-infrared results, we find that the fraction of objects with bonafide AGN is $25-30 \%$ for the 1 Jy sample of ULIGs and $35-50 \%$ for objects with $L_{\text {ir }}>10^{12.3} L_{\odot}$.

4. Comparisons of the dereddened emission-line luminosities of the optical or obscured BLRs detected in the ULIGs of the 1-Jy sample with those of optical quasars indicate that the obscured AGN/quasar in ULIGs is the main source of energy in at least $15-25 \%$ of all ULIGs in the 1-Jy sample. This fraction is closer to $30-50 \%$ among ULIGs with $L_{\text {ir }}>10^{12.3} L_{\odot}$.

These results are compatible with those from recent mid-infrared spectroscopic surveys carried out with ISO (e.g., Genzel et al. 1998). Indeed, a detailed object-by-object comparison of the optical and mid-infrared classifications shows an excellent agreement between the two classification methods (Lutz, Veilleux, \& Genzel 1999). These results suggest that strong nuclear activity, once triggered, quickly breaks the obscuring screen at least in certain directions, thus becoming detectable over a wide wavelength range.

\section{OPTICAL AND INFRARED IMAGING OF THE 1 JY SAMPLE}

We now have high signal-to-noise ratio, sub-arcsecond resolution $\mathrm{R}$ and $\mathrm{K}^{\prime}$ images of all ' $1 \mathrm{Jy}$ ' sources. Additionnal spectra were obtained of several sources in the field to identify them (stars versus galaxies) and determine if they are involved in the ULIG event. Our preliminary analysis of these data suggests the following tantalizing trends:

1. As found in previous studies, the large majority $(>95 \%)$ of the optical and near-infrared images show signs of a strong tidal interaction/merger in the form of distorted or double nuclei, tidal tails, bridges, and overlapping disks.

2. The small mean nuclear separation $(<3 \mathrm{kpc})$ of the ULIGs examined so far suggests that the majority of these galaxies are in a terminal stage of a merger. These mergers generally involve two large $\left(0.5-2 \mathrm{~L}^{*}\right)$ galaxies. Multiple mergers are seen in only 4 of the 118 systems. 
3. These galaxies span a broad range of total (= nuclear + host $)$ luminosities. In advanced mergers, $\mathrm{L}_{\mathrm{R}}($ tot $) \sim 1.5 \mathrm{~L}^{*}$ and $\mathrm{L}_{\mathrm{K}^{\prime}}($ tot $)$ $\sim 3 \mathrm{~L}^{*}$ on average but with a lot of scatter.

4. Roughly $30 \% / 10 \% / 60 \%$ of the R-band surface brightness profiles are well fitted by a elliptical-like $\mathrm{R}^{1 / 4}$-law / exponential disk / neither or both. The percentage of poor fits is likely to decrease when we examine our data at $\mathrm{K}^{\prime}$, where the effects of dust obscuration and star formation are less important.

5. Using a classification scheme first proposed by Surace (1998) and based on the results of published numerical simulations, we classify all our objects according to morphology: I. Pre-contact - relatively unperturbed and separate disk. II. First contact - overlapping disks but no evidence for strong bars or tidal tails. III. Pre-merger - two distinct galaxies with well-developed tidal tails and brigdes. (a) apparent separation $>10 \mathrm{kpc}$ and (b) apparent separation $<$ $10 \mathrm{kpc}$. IV. Merger - single nucleus with prominent tidal tails. (a) diffuse nucleus with $\mathrm{L}_{\mathrm{K}^{\prime}}(2 \mathrm{kpc}) / \mathrm{L}_{\mathrm{K}^{\prime}}($ tot $)<1 / 3$. (b) compact nucleus with $\mathrm{L}_{\mathrm{K}^{\prime}}(2 \mathrm{kpc}) / \mathrm{L}_{\mathrm{K}^{\prime}}$ (tot) $>1 / 3$. V. Old merger - no obvious signs of tidal tails but disturbed central morphology. We find that all Seyfert 1s and most of the Seyfert 2s are advanced mergers either based on their morphology (classes IVb or V; Fig. 1) or their nuclear separation ( $<5 \mathrm{kpc}$, generally; Fig. 2). A similar result is found when we consider the 'warm' objects with $f_{20} / f_{60}>0.2$ (Fig. 3).

\section{SUMMARY}

The results from our spectroscopic survey of the $1 \mathrm{Jy}$ sample of ULIGs indicate that the fraction of ULIGs powered by quasars increases with increasing infrared luminosity, reaching a value of $30-50 \%$ for $L_{\text {ir }}>10^{12.3} L_{\odot}$. The preliminary results from our imaging survey of the same sample suggest trends between merger phase, infrared colors, and the presence of an AGN. Objects with 'warm' quasar-like infrared colors show signs of AGN activity and are generally found in advanced mergers, based not only on the apparent nuclear separation but also on the morphology of the tidal tails. These results suggest that the evolutionary sequence 'cool' ULIGs $\rightarrow$ 'warm' ULIGs $\rightarrow$ quasars applies to at least some (though admittedly probably not all) ULIGs and quasars. 
Figure 1. Optical spectral type versus morphological class. See text for a description of the morphological classification.

Figure 2. Optical spectral type versus nuclear separation.

\section{Acknowledgments}

S. V. gratefully acknowledges the financial support of NASA through LTSA grant NAG 56547. 
Figure 3. IRAS $f_{20} / f_{60}$ colors versus morphological classification.

\section{References}

Genzel, R. et al. 1998, Ap. J., 498, 579

Kim, D.-C., \& Sanders, D. B. 1998, Ap. J. Suppl., 119, 41

Lutz, D., Veilleux, S., \& Genzel, R. 1998, Ap. J. (Letters), 517, L13

Sanders, D. B., \& Mirabel, I. F. 1996, AR\&A, 34, 725

Sanders, D. B., et al. 1988, Ap. J., 325, 74

Veilleux, S., Kim, D.-C., \& Sanders, D. B. 1999a, Ap. J., 522, 113

Veilleux, S., Sanders, D. B., \& Kim, D.-C. 1999b, Ap. J., 522, 139 


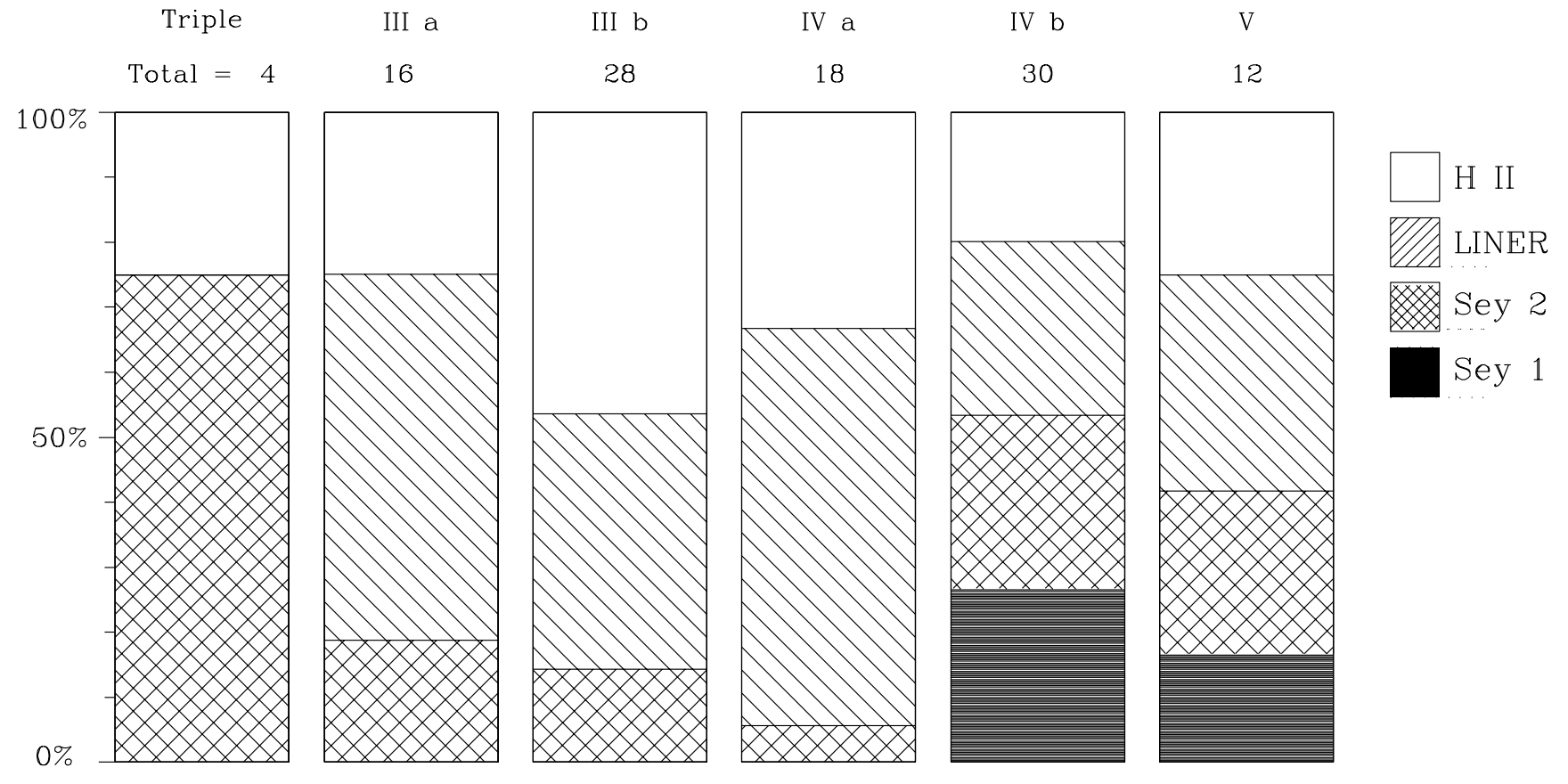




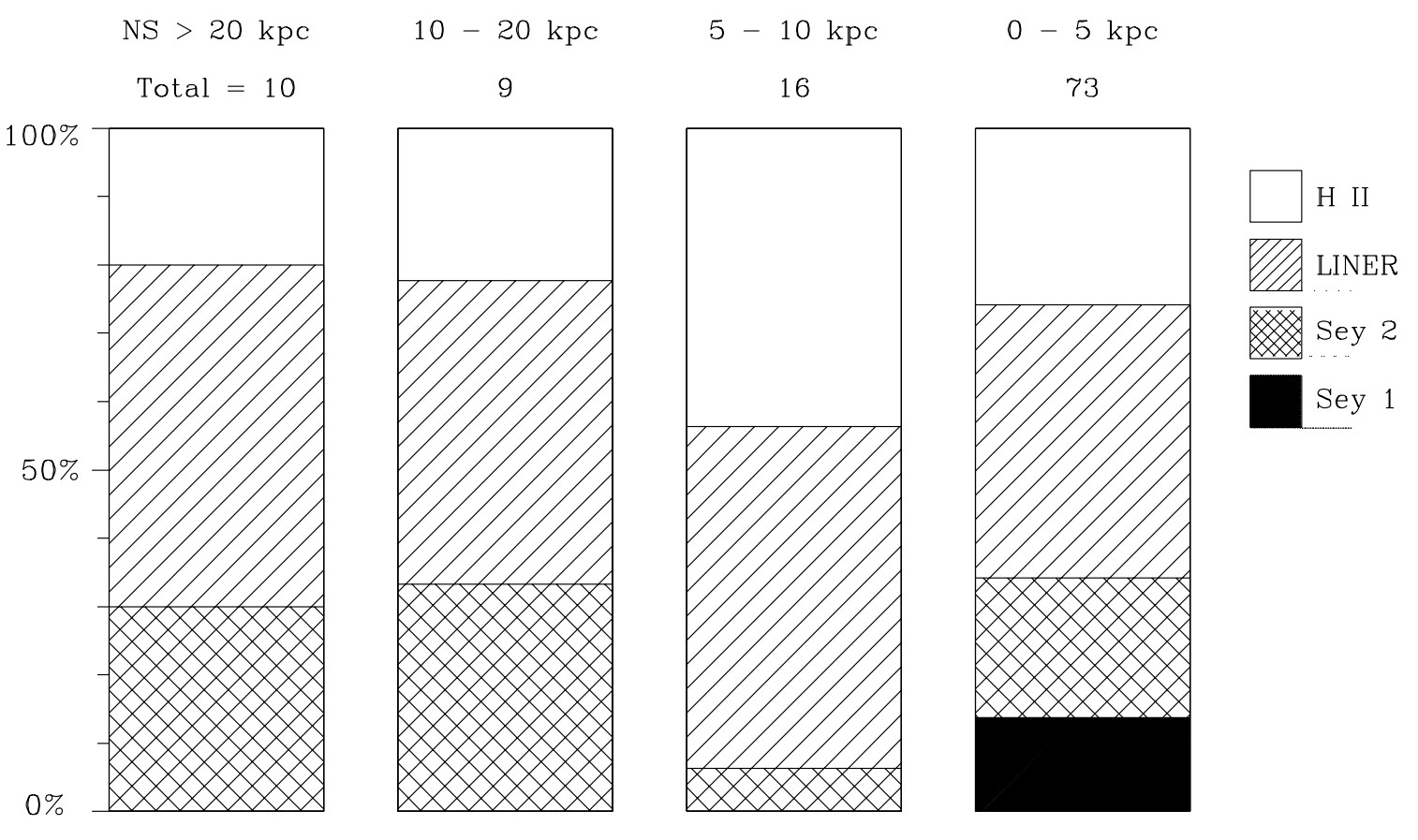


Triple

Total $=3$



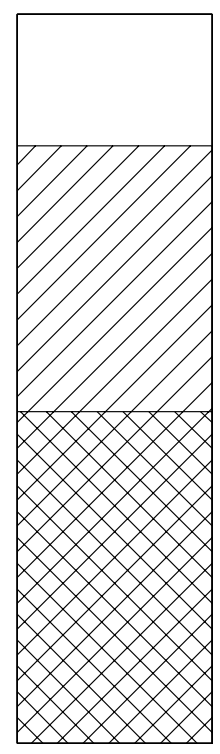

III $b$

21

IV a

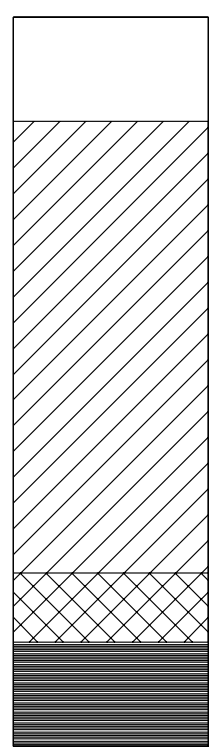

17

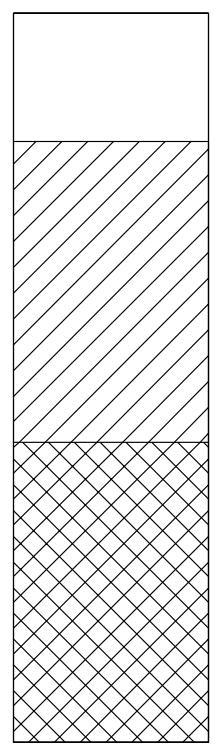

IV $\mathrm{b}$

27

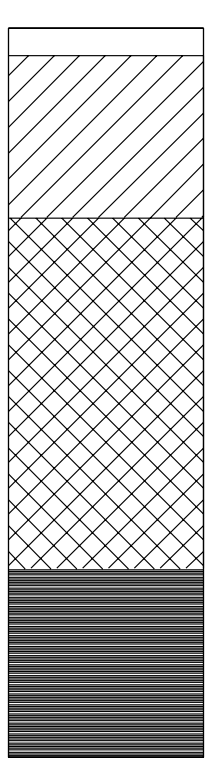

$\log ($ F25/F60)

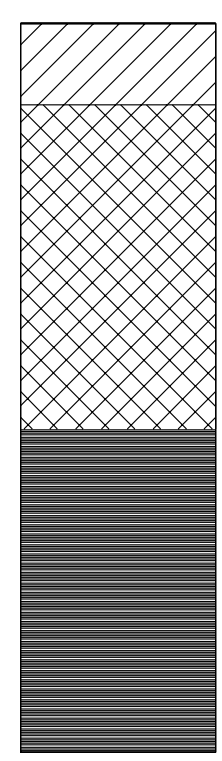

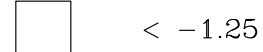

$\forall-0.95 \sim-1.25$

$-0.65 \sim-0.95$

$-0.35 \sim-0.65$ 